\title{
Medizinische Versorgungs-
} zentren

\section{- Eine innovative und attraktive Antwort auf die Heraus- forderungen an die ambulante Versorgung}

Mit dem GKV - Modernisierungsgesetz (GMG) von 2003 und dem Vertragsarztrechtsänderungsgesetz (VÄG) von 2006 hat der Gesetzgeber die Weichen für eine Flexibilisierung und Modernisierung der Rahmenbedingungen für die ambulante ärztliche Versorgung in Deutschland gestellt. Die möglichen Organisations- und Betriebsformen der ambulanten Versorgung wurden damit deutlich erweitert. Neben den traditionellen Kooperationsformen der Praxisgemeinschaft und der Gemeinschaftspraxis sind seit dem 1. Januar 2004 sogenannte Medizinische Versorgungszentren (MVZ) zur Teilnahme an der vertragsärztlichen Versorgung berechtigt. Der Koalitionsvertrag von CDU/CSU und FDP vom 26. Oktober 2009 sieht vor, die Voraussetzungen für Gründung und Betrieb eines solchen Zentrums einzuschränken. Der folgende Beitrag beleuchtet die qualitative und quantitative Entwicklung der neuen Versorgungsform und stellt sich kritisch gegen geplante Einschränkungen.

\section{Franz Knieps, Volker Amelung}

\section{Idee und Konzeption der Medizinischen Versorgungszentren}

Nach der Legaldefinition des § 95 Absatz 1 Satz 2 SGB V sind Medizinische Versorgungszentren fachübergreifende ärztlich geleitete Einrichtungen, in denen ins Arztregister eingetragene Ärzte als Angestellte oder als selbstständige Vertragsärzte tätig sind. Fachübergreifend ist eine solche Einrichtung immer dann, wenn dort Ärzte mit verschiedenen Facharzt- oder Schwerpunktbezeichnungen tätig sind. Gegründet werden können Medizinische Versorgungszentren von allen zugelassenen oder ermächtigten Leistungserbringern, also nicht nur von Vertragsärzten, sondern auch von Krankenhäusern, Apothekern, Physiotherapeuten und anderen Gesundheitsberufen. Krankenkassen oder sonstige Dritte - wie Managementgesellschaften oder private Investoren - sind nicht gründungsbefugt, können sich aber wirtschaftlich an Medizinischen Versorgungszentren beteiligen. Die Leitung muss stets durch einen Arzt oder eine Ärztin erfolgen. Solche Zentren dürfen sich aller zulässigen Rechtsformen bedienen, also auch der einer Kapitalgesellschaft. Medizinische Versorgungszentren unterliegen wie

Franz Knieps, Partner Wiese-Consult, Berlin

Prof. Dr. Volker Amelung, Medizinische Hochschule Hannover, Institut für Epidemiologie, Sozialmedizin und Gesundheitssystemforschung, Hannover andere Praxisformen auch der ärztlichen Bedarfsplanung. Damit unterliegen sie den gleichen Restriktionen in überversorgten Gebieten wie einzelne Niederlassungswillige.

Mit der Zulassung von Medizinischen Versorgungszentren in ganz Deutschland (In den neuen Bundesländern gewährleistete eine Übergangsbestimmung im Einigungsvertrag den (vorläufigen) Weiterbestand von Gesundheitszentren, die sich aus früheren Polikliniken entwickelt hatten.) wollte der Gesetzgeber

- einen organisatorischen Beitrag zur integrierten Versorgung ermöglichen,

- alternative Organisationsformen der ambulanten Versorgung zulassen und

- den Wettbewerb in der ambulanten Versorgung intensivieren.

Mit dem Vertragsarztrechtsänderungsgesetz aus dem Jahr 2006 hat der Gesetzgeber dann auch niedergelassenen Vertragsärzten eine Anstellung anderer Ärzte in unbegrenzter Zahl sowie das gleichzeitige Tätig werden im Krankenhaus und in der ambulanten Versorgung ohne die frühere Begrenzung auf eine belegärztliche Tätigkeit ermöglicht. Es war der erklärte Wille des Gesetzgebers, statt einer einzigen verbindlichen Vorgabe für alle ambulanten Versorgungsangebote die Organisations- und Betriebsformen nur den Beschränkungen zu unterwerfen, die sich aus den Sicher- 
heits- und Qualitätsanforderungen der medizinischen Versorgung ergeben.

Hierzu zählt unerlässlich die ärztliche Leitung jedes Zentrums. Selbst wenn ein anderer zugelassener Leistungserbringer ein MVZ gründen darf, die Leitung steht nur einem approbierten Arzt oder einer approbierten Ärztin zu. Die Freiberuflichkeit der ärztlichen Leitung und aller in einem Zentrum tätigen Ärztinnen und Ärzte, seien sie selbstständig oder angestellt, ist eine unverzichtbare Voraussetzung für Zulassung und Betrieb eines MVZ. Damit ist in gleicher Weise wie in anderen Betriebsformen sichergestellt, dass keine nichtärztlichen Interessen, wie beispielsweise das Streben nach einer hohen Kapitalrendite, die ärztliche Unabhängigkeit beeinträchtigen. Entsprechende Kritik an der Grundkonstruktion von Medizinischen Versorgungszentren mit externer Beteiligung ist ebenso unbelegt und substanzlos wie der Vorwurf der Ökonomisierung gegenüber Ärztinnen und Ärzten, die in anderen Betriebsformen tätig sind. Davon unberührt bleibt, dass der ärztlichen Leitung je nach Ausgestaltung der Innenverhältnisse wie im Krankenhaus auch medizinische Leitungsbefugnisse zustehen. Das gilt selbst für Vertragsärzte, die mit angestellten Ärzten in ihrer Praxis arbeiten. Und dies gilt grundsätzlich unabhängig von der Rechts-, Betriebs- oder Organisationsform, die für die jeweilige Einrichtung gewählt wird. Im Übrigen zeigt ein Blick auf die stationäre Versorgung, dass es gerade die privaten Krankenhausbetreiber sind, die die Qualitätssicherung und das Qualitätsmanagement durch zahlreiche Initiativen und Aktivitäten voran getrieben haben und treiben.

Auch der selbstständige Arzt wird in aller Regel - zumindest in den ersten Jahren und Jahrzehnten nach seiner Niederlassung - mit Fremdkapital arbeiten und auch später größere Investitionen in den medizinisch-technischen Fortschritt nur mit Hilfe von Fremdkapital tätigen können. Banken stellen Kredite nur dann zur Verfügung, wenn sichergestellt ist, dass der Kapitaldienst aus den Einnahmen der Praxis geleistet werden kann. Solches Fremdkapital muss verzinst (und getilgt!) werden wie die als Eigenkapital angelegten Fremdmittel privater oder institutioneller Anleger insbesondere bei Kapitalgesellschaften.

Bestimmte Bereiche und Schwerpunkte der spezialisierten ambulanten Medizin wie zum Beispiel die Labor- oder die Nuklearmedizin benötigen im Übrigen einen Kapitalaufwand, den einzelne Ärztinnen oder Ärzte kaum oder gar nicht mehr alleine aufnehmen können. Hier drängt sich die Gründung von Versorgungszentren unter Beteiligung externer Kapitalgeber geradezu auf. Ein hinreichender Grund zur Differenzierung der Gründungs- und Betriebsmöglichkeiten nach dem beruflichen Hintergrund des Gründers oder Eigentümers und/oder der Rechtsform, wie das die kassenärztlichen Körperschaften suggerieren, ist weder gesundheitspolitisch noch verfassungsrechtlich gegeben.

Schließlich zeigen auch die internationalen Erfahrungen, dass neue integrierte Organisationsformen - zum Beispiel in der Schweiz (z.B. medix), in Großbritannien (z.B. in Kooperation des National Health Service mit dem gemeinnützigen Managed Care Unternehmen Kaiser Permanente) oder in den USA (z.B. in Form der sogenannten Accountable Care Organizations) - im Hinblick auf die Herausforderungen der Zukunft entwickelt und erprobt werden.

\section{Die Entwicklung Medizinischer Versorgungszentren}

Seit 2004 steigt die Zahl neu gegründeter Medizinischer Versorgungszentren schnell an, wenngleich der absolute Anteil an der ambulanten Versorgung nach wie vor niedrig ist. Ihre Zahl ist Ende 2009 auf 1454 angewachsen. In diesen Zentren sind insgesamt 7127 Ärztinnen und Ärzte tätig, davon 1334 als Selbstständige und 5793 als Angestellte. Gerade in den letzten Jahren wächst der Angestelltenanteil deutlich schneller als die Zahl der Selbstständigen. Gleichwohl ist die überwiegende Zahl der Medizinischen Versorgungszentren in Trägerschaft von Vertragsärzten (49,5\%), während Krankenhäuser, die fast ausschließlich mit angestellten Ärzten arbeiten, 38,1\% der Zentren betreiben. MVZ werden sowohl in städtischen als auch in ländlichen Gebieten gegründet. Eine Mehrzahl der Zentren (rund $58 \%$ ) wird in Gebieten mit einer hohen Einwohnerzahl und Einwohnerdichte gegründet, während rund $42 \%$ im ländlichen Raum angesiedelt sind. Besonders auffällig ist, dass die meisten Zentren in Flächenländern wie Bayern, Nordrhein-Westfalen und Niedersachsen gegründet werden. Erst dann folgt der Stadtstaat Berlin. Allerdings weist die bayerische Landeshauptstadt München eine größere Zahl an MVZ auf als die Bundeshauptstadt. In all diesen Bundesländern sind MVZ überwiegend in der Hand von Vertragsärzten, während in Baden-Württemberg, Brandenburg, Mecklenburg-Vorpommern und Thüringen die Krankenhausträgerschaft vorherrscht. Trotz der Kritik mancher Hausärztefunktionäre sind die Hausärzte die am stärksten vertretene Arztgruppe in MVZ. Ihnen folgen Internisten, Chirurgen und Laborärzte, während Dermatologen, Reha-Mediziner und Urologen das Schlusslicht eines Arztgruppenvergleichs bilden. Die durchschnittliche Arbeitsgröße der Zentren steigt nur langsam auf nunmehr 4,9 Ärzte an. Als Rechtsform wird überwiegend die $\mathrm{GmbH}$ oder die Gesellschaft bürgerlichen Rechts gewählt. Echte Kapitalgesellschaften sind selten.

Die Entwicklung der letzten Jahre zeigt, dass sich unterschiedliche Geschäftsmodelle im Wettbewerb entwickelt haben:

- MVZ als Vertragspartner der Krankenkassen zur Umsetzung der populationsorientierten Integrationsversorgung. Gerade Medizinische Versorgungszentren mit einer ausreichenden Zahl von Ärztinnen und Ärzten entsprechender Breite der Fachrichtungen und differenzierter Kompetenz sind in der Lage, die Anforderungen an komplexe Verträge zu erfüllen. Das gilt speziell für Einrichtungen, die an verschiedenen Orten - über Filialen oder Franchising - tätig sind.

- MVZ als Einstieg für externe Investoren. Die ambulante Versorgung ist eine nachhaltige Anlagemöglichkeit, 
auch wenn diese nur eine begrenzte Rendite ermöglicht. MVZ ermöglichen den Aufbau von Organisationen, die das ausreichende Volumen für externes Kapital erreichen und über geeignete Gesellschafterstrukturen verfügen. Gerade die Rechtsform der Kapitalgesellschaft ermöglicht es ambitionierten Ärztinnen und Ärzten, aufwändige Investitionen über den Kapitalmarkt zu finanzieren.

- MVZ als Instrument zur Integration von ambulanter und stationärer Versorgung. Krankenhäuser erkennen in MVZ die Chance, ihren Anteil an der „Wertschöpfungskette“ zu sichern und angesichts der weiteren Verlagerung bisher stationär erbrachter Leistungen in die ambulante Versorgung auszuweiten. Damit besteht einerseits die Gefahr, dass es zu gesteuerten Einweisungen in Krankenhäuser des gleichen Eigentümers kommt. Andererseits bietet der Umbau der Strukturen auch die Chance, die notwendigen Strukturanpassungen in der stationären Versorgung zu beschleunigen und echte Integrationsversorgung anzubieten.

Die schnell wachsende Zahl von Medizinischen Versorgungszentren ist ein Beweis für die Attraktivität kooperativer ambulanter Versorgung sowohl bei Patientinnen und Patienten als auch bei Ärztinnen und Ärzten. Es bedarf kaum einer näheren Begründung, dass Patientinnen und Patienten eine Versorgung aus einer Hand bevorzugen und eine zwischen Ärzten verschiedener Fachgruppen sowie zwischen Ärzten und sonstigen Gesundheitsberufen koordinierte Behandlung wünschen, bei der es nicht zu unnötigen Wartezeiten, Doppeluntersuchungen, Medikationsfehlern und Reibungsverlusten zwischen unterschiedlichen Behandlern kommt.

Aber auch Ärztinnen und Ärzte haben das Bedürfnis zu organisierter Kommunikation und Kooperation mit ihren Kolleginnen und Kollegen sowie mit sonstigen Gesundheitsberufen. Dies gilt speziell für junge Ärztinnen und Ärzte, die gewohnt sind, im Team zu arbeiten und kooperative Führungsformen schätzen. Dabei ist auch zu berücksichtigen, dass sich der Anteil von Frauen in der Medizin kontinuierlich erhöht. Junge Medizinerinnen stellen - wie im Übrigen auch viele junge Mediziner - neue Anforderungen an die Vereinbarkeit von Familie und Beruf und an die Work-Life-Balance als frühere Arztgenerationen. Medizinische Versorgungszentren können hier besondere Arbeitsbedingungen, wie beispielsweise geregelte Arbeitszeiten, Teilzeitbeschäftigung oder Jobsharing, anbieten, die in kleineren Betriebsformen nur schwer oder gar nicht zu organisieren sind. Ohne solche Angebote besteht vermehrt die Gefahr, dass sich junge Mediziner und Medizinerinnen Arbeitsmöglichkeiten außerhalb der medizinischen Versorgung suchen.

\section{Versorgungspolitische Anforderungen an die ambu- lante Versorgung}

Aus versorgungspolitischer Sicht ist die bessere Verzahnung zwischen

- Haus- und Fachärzten,

- Fachärzten unterschiedlicher Fachrichtungen,

- Ärzten und sonstigen Gesundheitsberufen,

- ambulanter und stationärer Versorgung,

- Akutversorgung und Prävention,

- Rehabilitation und Pflege

notwendig, um die Bedürfnisse der Patientinnen und Patienten nach ganzheitlicher kontinuierlicher Versorgung zu befriedigen und einen gezielten Mitteleinsatz knapper Ressourcen ohne Verschwendung an den Schnittstellen zu gewährleisten. Die kontinuierliche Verlagerung der Versorgungsherausforderungen hin zu multimordid chronisch kranken Patienten erfordert gleichermaßen eine Anpassung der Versorgungsstrukturen. Sie können nur adäquat von multidisziplinären Teams versorgt werden, die zwingendermaßen und einer gemeinsamen elektronischen Patientenakte arbeiten. Gerade an den Schnittstellen entscheidet sich die Qualität einer organisierten Versorgung. Mängel hier spüren die Patientinnen und Patienten unmittelbar.

Medizinische Versorgungszentren sind ein wichtiges Instrument zur Vermeidung oder zum Abbau von Unterversorgung. Ihre innere Organisation lässt sich so flexibel gestalten, dass Satellitenpraxen eingerichtet oder Behandlungen an unterschiedlichen Orten angeboten werden.

Medizinische Versorgungszentren sind zudem ein geeignetes Instrument, Überkapazitäten in der stationären Versorgung abzubauen und den notwendigen Bettenabbau zu flankieren. Eingriffe, die noch vor wenigen Jahren nur stationär durchgeführt werden konnten, lassen sich heute überwiegend ambulant erbringen. Häufig bedarf es dazu allerdings (noch) einer gewissen Rückversicherung durch die Sicherheitsinfrastruktur eines kooperierenden Krankenhauses. Medizinische Versorgungszentren sind also ein wichtiges Bindeglied in der Verzahnung von ambulanter und stationärer Versorgung.

Medizinische Versorgungszentren sind eine Vorstufe zur umfassenden integrierten, patientenorientierten Gesundheitsversorgung. Dies gilt sowohl für die vertikale Integration über unterschiedliche Versorgungsebenen als auch für die horizontale Integration der Behandler, auch in interprofessioneller Kooperation. Eine Beschränkung der Betriebs- und Organisationsformen wäre ein Signal, die bisher im Konsens der meisten Akteure des Gesundheitswesens angestrebte Integration der Versorgung aufzugeben und zur zersplitterten Aufteilung in Sektoren und Subbereiche zurückzukehren. 


\section{Kritik an der Zulassung Medizinischer Versorgungszentren}

Obwohl sich Medizinische Versorgungszentren einer wachsenden Beliebtheit bei Ärzten und Patienten erfreuen, verstummt die Kritik aus Reihen der Ärzteschaft nicht. Sie kommt insbesondere aus der Politik und den Kassenärztlichen Vereinigungen. Soweit sie über die Sicherung bestehender Besitzstände hinaus geht, wird sie vor allem mit einer zunehmenden „Ökonomisierung“ des Gesundheitswesens durch externe Kapitalgeber begründet und mit ärztlicher Ethik und ärztlicher Berufsfreiheit gerechtfertigt. Eine solche Kritik unterschlägt, dass auch die ärztliche Einzelpraxis - speziell vor dem Hintergrund eines wachsenden Kapitalbedarfes - unter einem ebenso großen Wirtschaftlichkeitsdruck steht wie ein Medizinisches Versorgungszentrum. Sie übersieht, dass sozialrechtlich die ärztliche Leitung jedes Zentrums und berufsrechtlich die Therapiefreiheit gewährleistet ist. Argumente gegen eine „Ökonomisierung“ des Gesundheitswesens vernachlässigen schließlich, dass jede ärztliche Entscheidung auch eine Entscheidung über den Verbrauch von Ressourcen ist. Und Ressourcen sind - nicht nur im Gesundheitswesen - immer endlich.

Auch die Kritik an den Möglichkeiten für Krankenhäuser über Medizinische Versorgungszentren an der ambulanten Versorgung teilzuhaben, erweist sich bei näherem Hinsehen als interessengeleitet. Zum einen leisten angestellte Krankenhausärzte im Rahmen einer Ermächtigung einen wesentlichen Beitrag dazu, die ambulante Versorgung in der gewohnten Qualität sicherzustellen. Zum anderen ist die Verzahnung zwischen ambulanter und stationärer Versorgung erklärtes Ziel der Gesundheitspolitiker in wechselnden Regierungskoalitionen. Schließlich erweist sich auch die Kritik, Krankenhäuser würden sich über eigene MVZ die Einweisungen sichern, als wenig stichhaltig. Hierfür gibt es keine empirischen Belege. Das gilt im Übrigen auch für Vorwürfe, Versorgungszentren verzerrten den Wettbewerb zu Lasten niedergelassener Vertragsärzte in Einzelpraxen. Bei Belegärzten, die im Krankenhaus tätig werden, sind solche Entwicklungen nicht zu beobachten. Zudem wird jede Einweisung zur stationären Behandlung durch die zuständige Krankenkasse und gegebenenfalls auch den Medizinischen Dienst der Krankenversicherung geprüft. Schließlich dürfte der Reiz des Betriebs von MVZ durch Krankenhäuser eher in der umgekehrten Richtung liegen. Unter der Bedingung des DRG-Systems sind die Krankenhäuser gezwungen, die Verweildauer zu reduzieren und unnötige stationäre Kosten zu vermeiden. Das können sie erfolgreicher tun, wenn sie auf vor- und nachgelagerte Kapazitäten zurückgreifen können.

Nicht völlig von der Hand zu weisen sind zwei kritische Punkte, die immer wieder in Zusammenhang mit MVZ diskutiert werden, ohne dass es dafür aber bisher empirische Belege geben würde. So besteht auf Grund angebotsinduzierter Nachfrage die Gefahr der Überversorgung, wenn MVZ eine optimale Auslastung anstreben. Diese Gefahr kann allerdings mit einer populationsbezogenen Vergütung der Leistungen in MVZ begegnet werden. Zum an- deren besteht die theoretische Gefahr, dass bei der Umgestaltung der ambulanten Versorgungsstrukturen regionale Oligopole oder Monopole entstehen. Dieser Gefahr kann wirksam durch die Anwendung des Kartellrechts entgegen gewirkt werden. Dabei darf darauf aufmerksam gemacht werden, dass eine solche Entwicklung auch im Bereich der Krankenhäuser vorhergesagt wurde und bisher aber nur zu wenigen Eingriffen der Kartellbehörden geführt hat.

Insbesondere die Kritik der Kassenärztlichen Bundesvereinigung ist unverständlich, die mit ihren Forderungen ganz wesentlich die Handschrift des Koalitionsvertrags geprägt hat. Auch die KBV setzt richtigerweise auf die Zukunft ärztlicher Versorgungszentren mit angestellten Ärzten. Einzelne KV-en betreiben bereits eigene Praxen, um der Unterversorgung entgegen zu wirken. Die KBV gründete mit patiomed eine Aktiengesellschaft um ebenfalls eigene Zentren zu betreiben. Diese Entwicklung - losgelöst von rechtlichen Problemen die aus der ungeeigneten Rechtsform der KVen als Körperschaften des öffentlichen Rechts resultieren - ist grundsätzlich zu begrüßen und fördert den Wettbewerb um die geeignete Versorgungskonzepte und -strukturen.

Die Forderung allerdings, dass die Mehrheit der Anteile bei den involvierten Vertragsärzten liegen muss, ist nicht nur versorgungspolitischer Unsinn, sondern lädt auch zu Umgehungsversuchen ein. Wäre sie begründet, müsste sie auch für Krankenhäuser gelten.

Insgesamt finden sich bisher kaum Belege dafür, dass Kapitalgesellschaften wirtschaftlich motivierte Eingriffe in die ärztliche Freiberuflichkeit unternommen hätten. Dagegen gibt es viele Beispiele dafür, dass sich das Verhalten niedergelassener Ärzte primär am jeweiligen Honorierungssystem und nicht an den Notwendigkeiten der Versorgung ausrichtet. Trotzdem ist es nicht angebracht, daraus den Kampfbegriff „Ökonomisierung“ abzuleiten, der suggeriert, es gebe ein ärztliches Handeln außerhalb ökonomischer Gesetze.

\section{Zukunftsweisende Weiterentwicklung der Rahmenbedingungen}

Die Argumente für eine Beschränkung der Gründungsmöglichkeiten und der Eigentumsverhältnisse von Medizinischen Versorgungszentren sind nicht zielführend und wirken innovationshemmend. Sie sollen die niedergelassenen Ärzte unberechtigerweise vor Konkurrenz schützen (und zwar hinsichtlich der Versorgungsqualität, der Patientenpräferenzen und der Effizienz).

Eine wettbewerbs- und zukunftsorientierte Gesundheitspolitik muss

- sich an den Bedürfnissen der Patientinnen und Patienten orientieren und Vertrauen in deren Präferenzentscheidungen haben,

- eine kontinuierliche Behandlung mit möglichst wenig Schnittstellen gewährleisten, 
- den Ansprüchen und Wünschen künftiger Generationen von Ärztinnen und Ärzten gerecht werden,

- Kommunikation, Koordination und Kooperation zwischen Ärzten sowie zwischen Ärzten und sonstigen Berufsgruppen fördern,

- Organisationsvielfalt fördern, um unterschiedlichen Präferenzen gerecht werden zu können (angebots- und nachfrageseitig)

- die Investitionsbereitschaft in das Gesundheitswesen und speziell in die ambulante Versorgung stärken und

- den Wettbewerb zur Steigerung von Qualität und Wirtschaftlichkeit intensivieren.

All diese Anforderungen sprechen für einen weiteren Ausbau von Medizinischen Versorgungszentren und für pluralistische Eigentums-, Rechts-, Organisations- und Betriebsformen. Statt einer Eingrenzung der Möglichkeiten zum Betrieb von Medizinischen Versorgungseinrichtungen ist es geboten, diese durch den Ausbau der Selektivverträge im Wettbewerb um Qualität und Effizienz zu stärken. Auf Dauer bedarf es eines umfassenden Konzeptes zur wettbewerblichen Steuerung des Gesundheitswesens. Eine Regierung, die angetreten ist, die wettbewerbliche Ausgestaltung des Gesundheitswesens zu fördern, darf nicht zum Helfershelfer eines überholen Korporatismus werden und wettbewerbliche Anreize zurückführen, sondern muss das Vertrauen in innovative Ansätze stärken.

\section{Literatur}

Amelung / Cornelius, Wie beeinflussen Medizinische Versorgungszentren den Wettbewerb im Gesundheitswesen?, Public Health Forum 17 Heft $64 / 2009,23$ e

Amelung / Meyer-Lutterloh / Schmid / Seiler / Lägel / Weatherly, Integrierte Versorgung und Medizinische Versorgungszentren - Von der Idee zur Umsetzung, 2. Auflage, Berlin 2008

Braun / Güssow / Schumann / Heßbrügge (Hrsg.), Innovative Versorgungsformen im Gesundheitswesen - Konzepte und Praxisbeispiele erfolgreicher Finanzierung und Vergütung, Köln 2009

Dahm / Möller / Ratzel, Rechtshandbuch Medizinische Versorgungszentren, Berlin Heidelberg New York 2005

Dräger, Das Medizinische Versorgungszentrum am Krankenhaus, Stuttgart 2007

Hellmann / Eble / Kretzmann / Kurscheid, Medizinische Versorgungszentren erfolgreich führen und weiterentwickeln: Qualität steigern, Erträge ausbauen, Zukunft sichern, Berlin 2010

Isringhaus / Wendland, Medizinisches Versorgungszentrum - MVZ-kompakt, Erftstadt 2004

Kassenärztliche Bundesvereinigung (Hrsg.), Medizinische Versorgungszentren - Ein Leitfaden für die Praxis, 2. Auflage, Berlin 2009

Konerding, Der Vertragsarztsitz im Medizinischen Versorgungszentrum, Baden-Baden 2009

Schulte / Schulz, Medizinische Versorgungszentren: Verbesserung der ambulanten Patientenversorgung versus Selektion und Exklusion von Patientengruppen, Baden-Baden 2007

Wigge, Kooperationsformen im Vertragsarztrecht, In: Schnapp / Wigge (Hrsg.), Handbuch des Vertragsarztrechts - Das gesamte Kassenarztrecht, 2. Auflage, München 2006, 173 ff., insbesondere 190 ff.

Zwingel / Preißler, Das Medizinische Versorgungszentrum - Rechtliche Rahmenbedingungen für Gründung und Betrieb, Köln 2005

\section{Aktuelle}

Neuerscheinung

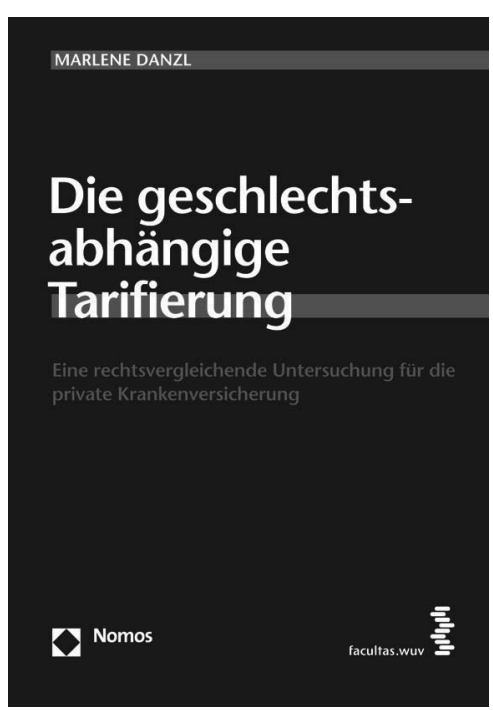

\section{Die geschlechtsabhängige Tarifierung}

Eine rechtsvergleichende Untersuchung für die private Krankenversicherung

Von Dr. Marlene Danzl

2010, 209 S., brosch., 32,- $€$,

ISBN 978-3-8329-5219-8

(Schriften zum Internationalen und

Vergleichenden Öffentlichen Recht, Bd. 12)

Aufgrund ihrer unterschiedlichen Schadenserwartung werden Frauen und Männer in der privaten Krankenversicherung sowohl in Deutschland als auch in Österreich unterschiedlichen Risikogruppen zugeordnet. Die Autorin prüft, ob diese geschlechtsabhängige Tarifierung mit Europa- und Verfassungsrecht vereinbar ist.

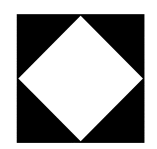

\section{Nomos}

Bitte bestellen Sie im Buchhandel oder versandkostenfrei unter $\checkmark$ www.nomos-shop.de 